\title{
Microperimetry as Part of Multimodal Assessment to Evaluate and Monitor Myopic Traction Maculopathy
}

This article was published in the following Dove Press journal: Clinical Ophthalmology

\author{
Pedro Manuel Baptista (D) \\ Nisa Silval \\ João Coelho (iD) \\ Diana José (D) \\ Daniel Almeida' \\ Angelina Meireles (iD) ${ }^{1,2}$ \\ 'Ophthalmology Department, Centro \\ Hospitalar Universitário do Porto, Porto, \\ Portugal; ' ${ }^{2}$ Instituto de Ciências \\ Biomédicas Abel Salazar, Porto, Portugal
}

Correspondence: Pedro Manuel Baptista Centro Hospitalar Universitário do Porto, Largo Prof. Abel Salazar, Porto 4099-00I, Portugal

Tel +35। 917868372

Email pedroyybaptista@gmail.com
Purpose: To characterize a population of high myopes with myopic traction maculopathy (MTM), to assess their retinal function, and to correlate it with anatomic status.

Patients and Methods: This was an observational cross-sectional study including 50 eyes from 27 patients. Demographic and clinical data were analyzed. Macular structure was assessed with spectral domain optical coherence tomography (SD-OCT, Heidelberg ${ }^{(\mathbb{B}}$ ) and macular function was studied with Microperimeter MP-3, NIDEK ${ }^{\circledR}$.

Results: The average for central foveal thickness (CFT) and choroid thickness (CT) was 213 $\pm 151 \mu \mathrm{m}$ and $36 \pm 23 \mu \mathrm{m}$, respectively, in a total of 50 eyes from 27 patients. In the microperimetry analysis, the average sensitivity on the foveal-centered $12^{\circ}$ polygon (CPS) was $14.37 \pm 9.1 \mathrm{~dB}$. CT was negatively associated with the bivariate contour ellipse areas (BCEA) $1(\mathrm{r}=-0.314 ; \mathrm{p}=0.034), 2(\mathrm{r}=-0.314 ; \mathrm{p}=0.034)$, and $3(\mathrm{r}=-0.316 ; \mathrm{p}=0.033)$. CPS had a strong positive correlation with best-corrected visual acuity (BCVA) $(r=0.661 ; p=0.000)$. We found a trend to worse microperimetric results in eyes with schisis $(n=19)(p>0.05)$ but eyes with atrophic areas $(n=33)$ presented significant inferior CPS $(p<0.001)$. The presence of staphyloma showed significant impact on macular sensitivities in eyes with areas of macular atrophy/fibrosis $(\mathrm{p}<0.05)$.

Conclusion: Macular microperimetry analysis can have a role as part of a multimodal anatomo-functional assessment for a more precise characterization of the high myopic patients with MTM, optimizing medical and surgical decisions.

Keywords: foveoschisis, high myopia, microperimetry, myopic traction maculopathy, retinal atrophy, staphyloma

\section{Introduction}

High myopia (HM) is associated with a refractive error of at least $-6 \mathrm{D}$ and/or an axial length $\geq 26 \mathrm{~mm}$. When accompanied by choroid and retinal degeneration with a variety of macular pathological changes is also named pathologic myopia (PM). ${ }^{1,2}$

Nowadays almost $50 \%$ of the world's population has myopia and, as it is increasing, the prevalence of HM is estimated to reach $10 \%$ worldwide in $2050 .^{3}$ It frequently presents a stable behavior, but progressive maculopathy may display a significant impact in visual function, frequently in active young patients, requiring vitreoretinal surgery. The pathological changes resulting from HM are already one of the main causes of serious visual impairment, even blindness, particularly in East Asian countries, like China, ${ }^{4}$ Singapore $^{5}$ or Japan, ${ }^{6}$ but also in Europe ${ }^{7,8}$ and 
United States. ${ }^{9}$ Therefore, HM is an emerging global health burden that urgently needs to be addressed.

Back in 2004, Panozzo et $\mathrm{al}^{10}$ proposed traction in the context of HM as the physiopathological basis for the subsequent chronic macular degeneration. Since then, the concept of "Myopic Traction Maculopathy" (MTM) has been subject of discussion and lack of consensus and efforts have been made in order to unify most of degenerative features generated by traction in the context of myopia under this entity. ${ }^{11}$ Nowadays, MTM is assumed as an umbrella term that encompasses a wide spectrum of related disorders, including staphyloma, vitreomacular traction (VMT), myopic foveoschisis, and myopic macular hole $(\mathrm{MH}){ }^{2}$

The prevalence of MTM is estimated to be between 9 and $34 \%$ of highly myopic eyes with posterior staphyloma ${ }^{10}$ Neither the pathogenesis nor the triggers of MTM development are completely understood, but there is consensus about the tríade of key factors driving the development of pathologic myopia: posterior staphyloma and elongation of the axial length, both associated with increased vitreous anteroposterior traction. ${ }^{2}$ However, tangential traction or remnants of the vitreous cortex, development of an epiretinal membrane, and inflexibility of retinal vessels are all thought to play a role. ${ }^{12}$ Additionally, it is believed today that the occurrence of staphyloma will, in most cases, eventually lead to the other full spectrum conditions of PM: chorioretinal atrophy and neovascular maculopathy. ${ }^{2}$

With the improvement of the image quality with Spectral Domain Optical Coherence Tomography (SDOCT), abnormal structures both in vitreoretinal and retinochoroidal interfaces are now readily analyzed. Given the complexity of this entity, recently, Medrano et $\mathrm{al}^{2}$ proposed a new full spectrum classification and grading system (ATN) for the myopic maculopathy which considers atrophic (A), tractional (T), and neovascular (N) components. This leads to a more precise staging of the disease, enabling clinicians to more accurately monitor changes over time. However, this classification does not encompass functional parameters like visual acuities or retinal sensitivities.

The emerging Microperimetry technology can translate macular functional status, by measuring retinal sensitivity on several points closely related to anatomic areas present in a fundus photography or OCT image. Additionally, it can have a direct role in treatments for MTM: the visual biofeedback training, based on a sensitivity characterization and subsequent education aimed to increase visual function using the less affected areas by macular degeneration has recently been shown as an option in various forms of maculopathy, including PM. ${ }^{13}$

The aim of the present study is to make a structural characterization of a population of high myopic patients with MTM, to assess their retinal function and correlate with anatomic status.

\section{Patients and Methods Design and Study Population}

Observational cross-sectional study. Inclusion criteria were: patients with diagnosed HM (<-6 diopters) and with different forms of MTM. Exclusion criteria were: presence of other macular or optic nerve diseases: presence of corneal alterations, cataract, or other ocular media hypo-transparency. The study adhered to the tenets of the Declaration of Helsinki. Approval was obtained from the "Departamento de Ensino, Formação e Investigação" (DEFI) from the Centro Hospitalar e Universitário do Porto, nr: 130-DEFI-132-CE. The informed consent from the patients was waived due to total anonymization and confidentiality of the data and the absence of detailed individual data.

\section{Demographic and Clinical Data}

All patients underwent a thorough ophthalmological evaluation, including slit lamp and mydriatic indirect fundoscopic exams. The best-corrected visual acuities (BVCA) were measured through a Snellen chart and converted to the logarithm of the minimum angle of resolution ( $\log M A R$ ) for statistical analysis. We analyzed objectively the lens status and the subjective refraction spherical equivalent (SE). Demographic data were collected, namely gender, age, the history of choroidal neovascularization (CNV), history of retinal detachment, or other concurrent ocular pathologies.

\section{Macular Structure Assessment}

The macular structure was assessed by SD-OCT, Heidelberg $^{\circledR}$. Only exams with good quality of acquisition were analyzed and we collected automated data from the central foveal thickness (CFT, central $1 \mathrm{~mm}$ circle) and manually measured subfoveal choroidal thickness (CT) without enhanced depth image (EDI) protocol. Within the $6 \times 6 \mathrm{~mm}$ area centered in the fovea we recorded:

- the presence of foveoschisis (T1, T2, and T3) and areas of external retinal atrophy or fibrosis (A2, A3, and A4), according to the newly proposed grading system for myopic maculopathy ${ }^{2}$ 
- the presence of vitreomacular traction (VMT) according to the International Vitreomacular Traction Study Group classification ${ }^{14}$

- the presence of posterior pole staphyloma. ${ }^{15}$

Eyes presented with myopic foveal macular hole in SDOCT (T4 and T5) ${ }^{2}$ were excluded.

\section{Macular Microperimetry Assessment}

The study of macular function was carried out with Microperimeter MP-3 (NIDEK $\left.{ }^{\circledR}\right)$. The exams were performed in a 31.4 asb white background, with a Goldmann $\mathrm{V}$ white stimulus (34 $\mathrm{dB}$ dynamic range) through a fast (4-2) strategy and a single cross red $\left(2^{\circ}\right)$ fixation target. We only analyzed reliable exams. Data was collected from the mean retinal sensitivity of the 24 measured points in the fovealcentered $12^{\circ}$ polygon (CPS) and from the fixation capacity of the eye through the analysis of the fixation stability (\% of fixations within the $2^{\circ}\left(\mathrm{F}^{\circ}\right)$ and $4^{\circ}\left(\mathrm{F}^{\circ}\right)$ foveal centered circles), and the bivariate contour ellipse areas of the 1 (68.2\%), 2 (95.4\%), and 3 (99.6\%) standard deviations (SD) of the total fixations (BCEA 1, 2, and 3, respectively).

\section{Statistical Analysis}

Statistical analysis was performed with the SPSS statistical software package, version 24.0 (SPSS ${ }^{\circledR}$, Chicago, IL). Normality of the data was confirmed by the ShapiroWilk test. Levene's test was used to look for homogeneity of variances, and Student's $t$-test was used to compare variables between groups. When nonparametric tests were needed, the Wilcoxon rank-sum test was applied. Possible correlations were studied with Spearman rank correlation method. Values are shown as mean \pm standard deviation unless otherwise specified. All p-values (p) were 2 -sided, and $p$-values $<0.05$ were considered significant.

\section{Results}

\section{General Analysis - First Step}

The first step of the analysis was an anatomical and functional characterization of all samples.

Fifty eyes from 27 patients were analyzed. The sample was constituted by 6 men and 21 women, aged $57.9[34 ; 81]$ years old. The mean SE was $-15.6 \pm 6.6$ diopters. Regarding the forms of MTM, we found some degree of foveal retinal splitting/schisis in 19 (38\%) eyes, areas of external retina atrophy or fibrosis in $33(66 \%)$ eyes, VMT in $9(18 \%)$ eyes, and posterior pole staphyloma in $31(62 \%)$ eyes (Figure 1).

Overall, we found a mean CFT of $216.7 \pm 142 \mu \mathrm{m}$ and a mean $\mathrm{CT}$ of $49.1 \pm 55 \mu \mathrm{m}$ (Table 1 ). In the microperimetry exam, we found an overall average sensitivity on the CPS of $14.4 \pm 9.1 \mathrm{~dB}$, below the normal range. Stable fixation was achieved by $62 \%(\mathrm{n}=31)$ of the eyes, with overall averages of $78.4 \pm 23 \%$ in the $\mathrm{F}^{\circ}$ and $92.8 \pm 14 \%$ in the $\mathrm{F} 4^{\circ}$ (Table 1). The overall BCEA1, 2, and 3 were $3.5 \pm 5.8^{\circ}$, $9.3 \pm 15.6^{\circ}$, and $17.9 \pm 29.7^{\circ}$, respectively (Table 1 ).

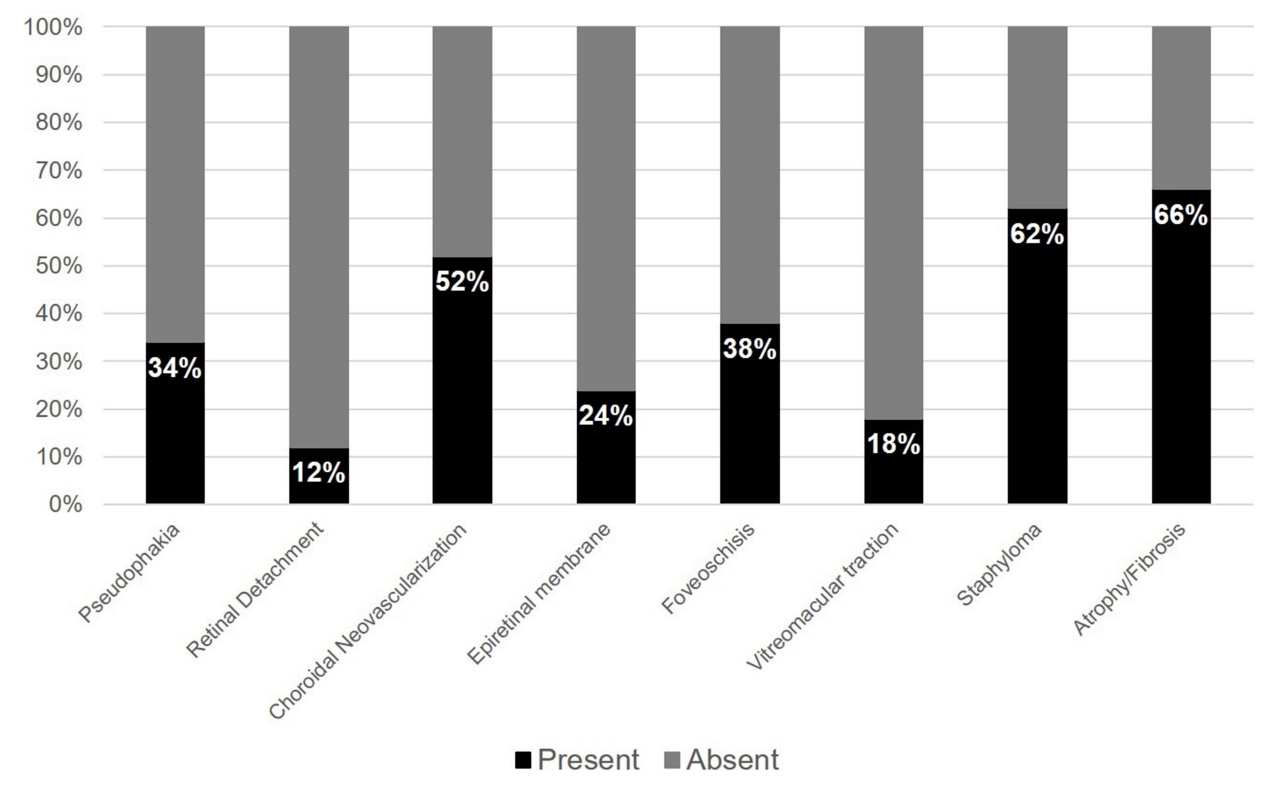

Figure I Ophthalmic characterization, $\%$ ( $n=50$ eyes). 


\section{Subgroup Analysis - Second and Third Steps}

The subgroup analysis was made through two steps:

- Second step: Two independent comparative analysis on anatomic and microperimetric data: first, comparing the group of eyes with some degree of retinal splitting/ schisis (foveoschisis group, $n=19$ ) with those without this alteration and second comparing the group of eyes with some degree of fibrotic/atrophic areas in the outer retina (atrophy/fibrosis group, $\mathrm{n}=33$ ) with those without this alteration (Table 1).

- Third step: Stratification of both the foveoschisis group ( $n=19$, Table 2) and the atrophy/fibrosis group $(n=33$, Table 3$)$ for the presence of either anterior traction (VMT) or posterior traction (Staphyloma) components.

\section{Foveoschisis Group}

Within the eyes with foveoschisis, 6/19 had concomitantly important VMT, 13/19 had staphyloma and 12/19 had areas of retinal atrophy/fibrosis.

The patients in this group were non-significantly older $(p=0.147)$ and these eyes had non-significantly lower BCVA $(p=0.753)$ and SE $(p=0.608)$. The CFT was significantly higher $(\mathrm{p}=0.038)$ and the CT was nonsignificantly lower $(\mathrm{p}=0.283)$ (Table 1$)$.

In the microperimetric analysis, we found in this group non-significantly inferior CPS $(p=0.973)$ and fixation stabilities either in $\mathrm{F} 2^{\circ}(\mathrm{p}=0.902)$ and $\mathrm{F} 4^{\circ}(\mathrm{p}=0.593)$. Additionally, the BCEA1 $(p=0.858)$, BCEA2 $(p=0.858)$, and BCEA3 $(\mathrm{p}=0.867)$ were non-significantly larger (Table 1$)$.

In the stratified analysis within this group, either the eyes presenting VMT and those with staphyloma had nonsignificantly lower BCVA ( $p>0.05$ ). We found significantly higher CFT in the VMT subgroup $(\mathrm{p}=0.031)$. Regarding microperimetric measurements, the eyes with staphyloma showed non-significantly worse values both in retinal sensitivities and fixation areas ( $\mathrm{p}>0.05$ in all) (Table 2 ).

\section{Atrophy/Fibrosis Group}

Within the eyes with external retina atrophy/fibrosis, 21/33 had concomitant staphyloma, 12/33 had foveoschisis and 6/33 had VMT.

The patients in this group were non-significantly older $(p=0.134)$ and with non-significantly higher SE $(p=0.245)$. We found marked alterations either in functional and anatomical outcomes in these eyes: significantly lower BCVA $(p=0.007)$, CPS $(p<0.001)$, CFT $(p=0.088)$, and CT $(\mathrm{p}=0.027)$ (Table 1).

Table I Overall and per Group Analysis

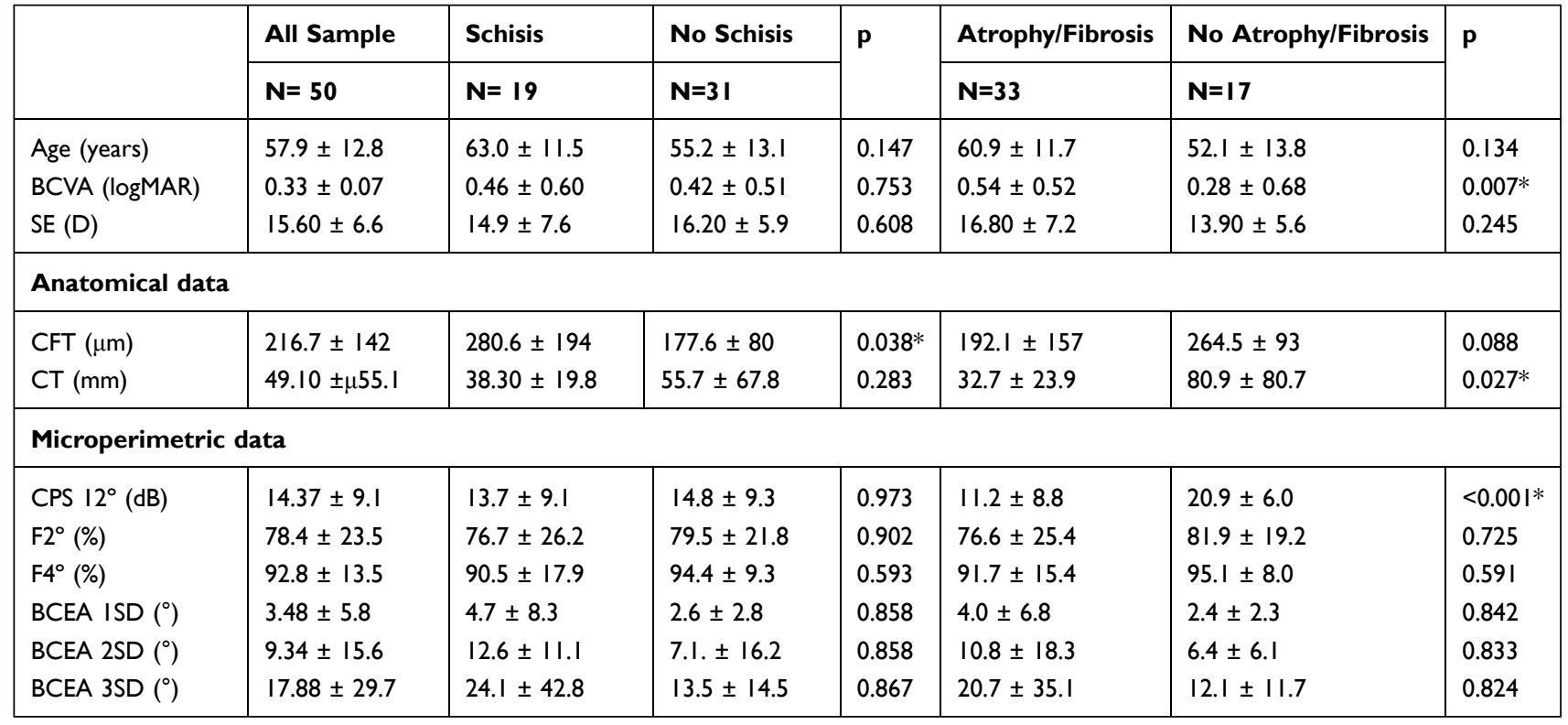

Note: *Statistical significance for a $p<0.05$.

Abbreviations: BCVA, best-corrected visual acuity; SE, spherical equivalent; CFT, central Imm foveal thickness; CT, subfoveal choroidal thickness; CPS I2, retinal sensitivity in the foveal centered $12^{\circ}$ polygon; $\mathrm{F} 2$ and F4, proportion of fixations within the $2^{\circ}$ and $4^{\circ}$ foveal centered circles; BCEA ISD, 2SD and 3SD, bivariate contour ellipse areas of the I (68.2\%), 2 (95.4\%) and $3(99.6 \%)$ standard deviations of the total fixations. 
Table 2 Subgroup Analysis Within Eyes with Schisis

\begin{tabular}{|c|c|c|c|c|c|c|}
\hline & \multirow{2}{*}{$\begin{array}{l}\text { VMT } \\
N=6\end{array}$} & \multirow{2}{*}{$\begin{array}{l}\text { No VMT } \\
\mathrm{N}=13\end{array}$} & \multirow[t]{2}{*}{$\mathbf{p}$} & \multirow{2}{*}{$\begin{array}{l}\text { Staphyloma } \\
N=13\end{array}$} & \multirow{2}{*}{$\begin{array}{l}\text { No Staphyloma } \\
N=6\end{array}$} & \multirow[t]{2}{*}{$\mathbf{p}$} \\
\hline & & & & & & \\
\hline Age (years) & $64.3 \pm 7.1$ & $62.33 \pm 13.8$ & 0.782 & $64.3 \pm 11.8$ & $60.3 \pm 12.9$ & 0.654 \\
\hline BCVA (logMAR) & $0.51 \pm 0.77$ & $0.43 \pm 0.54$ & 0.651 & $0.54 \pm 0.70$ & $0.33 \pm 0.52$ & 0.175 \\
\hline SE (D) & $21.13 \pm 8.9$ & $12.11 \pm 5.4$ & 0.042 & $15.42 \pm 8.8$ & $13.69 \pm 4.7$ & 0.722 \\
\hline \multicolumn{7}{|c|}{ Anatomical data } \\
\hline $\mathrm{CFT}(\mu \mathrm{m})$ & $418.0 \pm 282$ & $217.2 \pm 97$ & $0.031 *$ & $307.9 \pm 217$ & $221.5 \pm 127$ & $0.38 I$ \\
\hline CT (mm) & $36.00 \pm 27.1$ & $39.38 \pm 16.7$ & 0.579 & $43.31 \pm 21.2$ & $27.50 \pm 11.5$ & 0.087 \\
\hline \multicolumn{7}{|c|}{ Microperimetric data } \\
\hline $\mathrm{CPS} 12^{\circ}(\mathrm{dB})$ & $15.46 \pm 9.2$ & $12.92 \pm 9.4$ & 0.586 & $12.35 \pm 9.7$ & $16.70 \pm 7.8$ & 0.348 \\
\hline $\mathrm{F} 2^{\circ}(\%)$ & $74.3 \pm 18.0$ & $77.9 \pm 29.9$ & 0.282 & $71.2 \pm 29.9$ & $88.7 \pm 9.4$ & 0.210 \\
\hline $\mathrm{F} 4^{\circ}(\%)$ & $93.3 \pm 7.3$ & $89.2 \pm 21.3$ & 0.579 & $87.1 \pm 20.9$ & $98.0 \pm 2.0$ & 0.323 \\
\hline BCEA ISD $\left(^{\circ}\right)$ & $3.42 \pm 2.3$ & $5.26 \pm 10.0$ & 0.282 & $6.12 \pm 9.8$ & $1.57 \pm 1.0$ & 0.323 \\
\hline BCEA 2SD $\left({ }^{\circ}\right)$ & $9.17 \pm 6.3$ & $14.18 \pm 26.9$ & 0.282 & $16.47 \pm 23.3$ & $4.20 \pm 2.5$ & 0.323 \\
\hline BCEA 3SD $\left({ }^{\circ}\right)$ & $17.55 \pm 12.1$ & $27.1 \pm 51.5$ & 0.323 & $31.52 \pm 50.4$ & $8.00 \pm 5.0$ & 0.323 \\
\hline
\end{tabular}

Note: *Statistical significance for a $p<0.05$.

Abbreviations: BCVA, best-corrected visual acuity; SE, spherical equivalent; CFT, central Imm foveal thickness; CT, subfoveal choroidal thickness; CPS $12^{\circ}$, retinal sensitivity in the foveal centered $12^{\circ}$ polygon; F2 and F4, proportion of fixations within the $2^{\circ}$ and $4^{\circ}$ foveal centered circles; BCEA ISD, 2SD and 3SD, bivariate contour ellipse areas of the I (68.2\%), 2 (95.4\%) and 3 (99.6\%) standard deviations of the total fixations.

Table 3 Subgroup Analysis Within Eyes with Areas of Macular Atrophy/Fibrosis

\begin{tabular}{|c|c|c|c|c|c|c|}
\hline & VMT & No VMT & \multirow[t]{2}{*}{$\mathbf{p}$} & Staphyloma & No Staphyloma & \multirow[t]{2}{*}{$\mathbf{p}$} \\
\hline & $N=6$ & $\mathbf{N}=\mathbf{2 7}$ & & $\mathbf{N}=\mathbf{2} \mathbf{I}$ & $N=\mid 2$ & \\
\hline Age (years) & $70.3 \pm 11.6$ & $58.8 \pm 11.0$ & 0.127 & $62.9 \pm 11.6$ & $56.6 \pm 12.00$ & 0.335 \\
\hline BCVA (logMAR) & $0.72 \pm 0.80$ & $0.51 \pm 0.50$ & 0.360 & $0.72 \pm 0.68$ & $0.34 \pm 0.46$ & $0.030 *$ \\
\hline SE (D) & $18.33 \pm 14.6$ & $16.50 \pm 5.5$ & 0.849 & $17.16 \pm 8.2$ & $16.21 \pm 5.36$ & 0.803 \\
\hline \multicolumn{7}{|c|}{ Anatomical data } \\
\hline $\mathrm{CFT}(\mu \mathrm{m})$ & $383.8 \pm 306$ & $149.5 \pm 46$ & $<0.00 I^{*}$ & $210.2 \pm 193$ & $160.5 \pm 50$ & 0.391 \\
\hline CT $(\mathrm{mm})$ & $29.67 \pm 20.5$ & $33.37 \pm 24.9$ & 0.803 & $29.52 \pm 16.6$ & $38.25 \pm 33.4$ & 0.087 \\
\hline \multicolumn{7}{|c|}{ Microperimetric data } \\
\hline CPS $12^{\circ}(\mathrm{dB})$ & $10.70 \pm 8.2$ & $11.34 \pm 9.0$ & 0.885 & $8.29 \pm 7.7$ & $16.59 \pm 8.4$ & $0.009 *$ \\
\hline $\mathrm{F} 2^{\circ}(\%)$ & $58.2 \pm 29.5$ & $80.2 \pm 23.5$ & 0.115 & $72.6 \pm 26.0$ & $83.9 \pm 23.5$ & 0.210 \\
\hline $\mathrm{F} 4^{\circ}(\%)$ & $85.6 \pm 13.4$ & $92.9 \pm 15.8$ & 0.129 & $89.6 \pm 17.5$ & $95.6 \pm 10.3$ & 0.323 \\
\hline BCEA ISD $\left({ }^{\circ}\right)$ & $5.8 \pm 4.6$ & $3.7 \pm 7.2$ & 0.091 & $4.9 \pm 8.1$ & $2.4 \pm 3.5$ & 0.323 \\
\hline BCEA 2SD $\left({ }^{\circ}\right)$ & $15.7 \pm 12.4$ & $9.9 \pm 19.3$ & 0.091 & $13.2 \pm 21.6$ & $6.5 \pm 9.5$ & 0.323 \\
\hline BCEA 3SD $\left({ }^{\circ}\right)$ & $30.0 \pm 23.7$ & $18.9 \pm 37.0$ & 0.091 & $25.2 \pm 41.4$ & $12.4 \pm 18.2$ & 0.323 \\
\hline
\end{tabular}

Note: *Statistical significance for a $p<0.05$.

Abbreviations: BCVA, best-corrected visual acuity; SE, spherical equivalent; CFT, central Imm foveal thickness; CT, subfoveal choroidal thickness; CPS I $2^{\circ}$, retinal sensitivity in the foveal centered $12^{\circ}$ polygon; F2 and F4, proportion of fixations within the $2^{\circ}$ and $4^{\circ}$ foveal centered circles; BCEA ISD, 2SD and 3SD, bivariate contour ellipse areas of the I (68.2\%), $2(95.4 \%)$ and $3(99.6 \%)$ standard deviations of the total fixations.

In the remaining microperimetric analysis we found non-significantly lower fixation stability in the $F 2^{\circ}$ $(\mathrm{p}=0.725)$ and $\mathrm{F} 4^{\circ}(\mathrm{p}=0.591)$ and non-significantly larger BCEA1 $(p=0.842), \quad$ BCEA2 $(p=0.833)$ and BCEA3 $(\mathrm{p}=0.824)($ Table 1$)$.
In the stratified analysis within this group, the eyes presenting with staphyloma had significantly lower BCVA $(p=0.030)$. Regarding anatomical analysis, the VMT subgroup showed significantly higher CFT $(p<0.001)$. On the microperimetric assessment we found 
significantly lower CPS in eyes with staphyloma $(p=0.009)$ and non-significantly lower $\mathrm{F}^{\circ}$ and $\mathrm{F} 4^{\circ}(\mathrm{p}>0.05$ in all) and larger BCEA's ( $>0.05$ in all) in each subgroup (Table 3 ).

\section{Correlations - Fourth Step}

We studied possible correlations regarding all eyes in our sample. Age was negatively associated with CPS ( $\mathrm{r}=$ $-0.500 ; \mathrm{p}=0.013$ ). Regarding anatomical parameters, CFT was positively associated with BCVA $(r=0.322 ; \mathrm{p}=0.023)$. Besides, CT was positively associated with CFT ( $\mathrm{r}=0.429$; $\mathrm{p}=0.034$ ) and was the anatomic parameter more closely associated with the microperimetric values with negative correlations with the BCEA1 ( $\mathrm{r}=-0.314 ; \mathrm{p}=0.034)$, BCEA2 ( $\mathrm{r}=-0.314 ; \mathrm{p}=0.034)$ and BCEA3 $(\mathrm{r}=-0.316$; $\mathrm{p}=0.033$ ). The only microperimetric parameter associated with BCVA was the CPS, with a strong positive correlation $(\mathrm{r}=0.661 ; \mathrm{p}<0.001)$.

\section{Discussion}

As this study was made in a tertiary center, we found high prevalence of structural high-grade abnormalities, like $\mathrm{CNV}$ and posterior pole atrophy or fibrosis, which could lead to worse functional results. Nevertheless, the prevalence of both foveoschisis, VMT, and staphyloma are similar to those reported in the literature. ${ }^{2}$

Another difficulty in the management of these patients is the challenge to detect the visual damage in HM with high sensitivity, as the progression of HM and related pathological changes is slow and visual acuity might be a poor functional endpoint as it may be unaffected until late stages of MTM. ${ }^{16}$ In contrast, microperimetry technology has the potential of a more accurate monitoring of HM-related visual damage in the macular area, measuring the visual function by projecting a localized visual stimulus directly onto exact positions. ${ }^{17-19}$ Recently, Wang et al ${ }^{20}$ highlighted this idea, showing that several external retina characteristics, namely the cone density and morphology and the thickness of the myoid and ellipsoid zones can be significant predictors of microperimetric results. The same study outpointed that early microperimetric monitoring can be an important tool to develop protocols that will help evaluate and prevent further visual impairment in HM. However, as far as the authors knowledge, only one study described microperimetric results in the subset of $\mathrm{MTM}^{21}$ reporting an increase in both BCVA and macular sensitivities after cataract surgery. In the present study, a strong positive correlation was found between BCVA and CPS values. Additionally, significantly lower sensitivities were shown in eyes with atrophy/fibrosis and, within this group, in those with staphyloma. This highlights the role of microperimetry in the assessment of differential functional loss associated with the different morphological alterations found in MTM.

Myopic foveoschisis involves a progressive separation of retinal layers, which remain connected by Müller cells. Although considered to be a slowly progressive-condition, it may lead to foveal detachment and full-thickness MH in more severe cases. ${ }^{22}$ Regarding the eyes with schisis, we found an expected significant higher CFT, as the retina is split but only slightly worse BCVA and microperimetric results and this is in line with the reported relative benignity of this alteration. In fact, Shimada et $\mathrm{al}^{23}$ reported variations in the natural course of myopic retinoschisis in 207 eyes during 3 years and showed that only the full 6-mm foveal-centered area of retinoschisis (S4) was associated with high probability - $43 \%$ - of progression in comparison to only $3.6 \%$ in the $\mathrm{S} 1$ eyes, $8.9 \%$ in the $\mathrm{S} 2$ eyes, and $13.0 \%$ in the S3 eyes. Additionally, they observed that nearly $11 \%$ of those S4 eyes improved without treatment during the 3 years follow up. This can be due to the absorption by a young retinal pigment epithelium pump, restoring the retinal anatomy. In the present study, within the foveoschisis group, we further studied separately the subgroups of eyes with VMT and those with staphyloma. Although none statistically significant difference was found in each stratified analysis, eyes with VMT showed non-significantly better microperimetric results, while eyes with staphyloma showed non-significantly worse microperimetric results. This highlights the idea that when schisis is present, the major factor of functional impairment can be the posterior traction component instead of the more benign anterior VMT-associated retinal thickness increase by itself. Therefore, the microperimetric analysis can be added to the visual acuity evaluation and enhance the sensitivity to detect functional loss.

Thinning of the choroid in myopia and its relationship with visual function have been widely studied. In the present study, the mean CT in all sample was lower than the reported ${ }^{18,24,25}$ and this can be due to the absence of EDI protocol. Additionally, it was reported that significant degeneration of choroidal perfusion occurs in high myopia, which may lead to photoreceptor dysfunction ${ }^{26}$, and nowadays it is even defended the role of the choroid homeostasis as a potential causative factor for the development and progression of PM alterations. ${ }^{27}$ When we analyze the group of eyes with areas of macular atrophy/ 
fibrosis, besides the expected functional worse results in BCVA and the retinal sensitivities (CPF), we found cleared diminished anatomical parameters, mainly the $\mathrm{CT},{ }^{28}$ which was the parameter, in general, more associated with microperimetric results. Therefore, we can infer the possible prognosis role of this technology in these patients. Beyond that, if we look for the subgroup analysis within these eyes with atrophic changes, we found: first, the eyes with staphyloma were associated to a worse retinal sensitivity result; second, the eyes with VMT showed higher retinal thickness and the worst fixation results in the microperimetric analysis. This is in line with the reported idea $^{29}$ that the VMT, mainly associated with posterior pole staphyloma, can be the trigger to the detachment of the foveal photoreceptors and subsequent functional loss and our results can highlight the potential role of fixation capability assessment in monitoring patients with VMT associated to a greater degeneration of the external retina and choroid.

Our findings are in line with the reported in literature and corroborate two ideas: first, the general benignity of the retinal split in the PM; second, the VMT acting as a trigger to progression of visual impairment in eyes with less functional reserve. As the staphyloma component is present in many of PM eyes, encompassing many of the other alterations concomitantly, it is more difficult to study this posterior globe bulging component repercussion alone but it showed to be the main factor linked to more severe chorioretinal degeneration in this study.

If we aggregate all this results within an anatomofunctional approach instead of the classical anatomicbased view, our work highlights two main ideas. One, that in schitic eyes the main factor associated with functional loss and possibly progression may be the presence of staphyloma instead of the VMT itself and the microperimetric analysis can help to better monitor which of those eyes are losing retinal function and probably progressing to macular hole stage and so can gain with surgical intervention before the schisis progresses. Two, that in the eyes with greater degeneration of the external retinal layers, both the sensitivity and the fixation capability assessments can be also of value by addressing the true repercussion of the anterior VMT as a real trigger to functional decrease and even being a basis for the proper utilization of visual biofeedback techniques in order to improve their quality of life. Thus, a microperimetry-based protocol can be of value to conduct early intervention in
Table 4 Functional Severity Scale

\begin{tabular}{|l|l|}
\hline Grade & Macular Morphologic Alterations in Eyes with MTM \\
\hline $\mathbf{I}$ & Foveoschisis \pm vitreomacular traction \\
$\mathbf{2}$ & Foveoschisis + staphyloma \\
$\mathbf{3}$ & Outer retina atrophy \\
$\mathbf{4}$ & Outer retina atrophy + staphyloma \\
$\mathbf{5}$ & Outer retina atrophy + vitreomacular traction \\
\hline
\end{tabular}

Abbreviation: MTM, myopic traction maculopathy.

order to protect the photoreceptors and enhance visual function in $\mathrm{HM}$ patients. The authors propose a functional severity scale according to microperimetric results and including the aforementioned conclusions regarding the different morphologic alterations in MTM (Table 4).

To our knowledge, this study is the first study trying to make a comprehensive anatomical and functional characterization of patients with different pathologic myopia retinal alterations and encompassing the most recent microperimetry technology. As this multimodal assessment can have a role in many ways, like the staging and prognosis both in the difficult surgical decisions and even in conservative approaches like visual biofeedback training, the authors consider the subgroup analysis a major strength, even without the possibility of studying each retinal alteration alone.

The authors consider disadvantages and limitations of this study: cross-sectional nature; lack of HM non-PM control group; lack of an "ILM peeled" group; CT measurements without EDI protocol. On the other hand, we don't consider the manual CT measurements a limitation, as the automatic segmentation has very low reproducibility in these patients.

\section{Conclusion}

Despite the wide presentation and severity spectrum of MTM, it withstands a great impact on the visual function of active patients and due to the increasing incidence of HM worldwide, the prevalence of alterations associated to PM will be a public health problem. Regarding the structural singularity of this population, anatomic assessment is limited by the absence of a normative basis, which unable effective monitoring of disease progression. Our study highlights the role of microperimetry analysis as part of a multimodal anatomo-functional assessment for a more precise characterization of these patients, optimizing medical and surgical decisions. 


\section{Acknowledgments}

The authors want to acknowledge all the support granted by the head of the Ophthalmology department of Centro Hospitalar e Universitário do Porto, Prof. Dr. Pedro Menéres.

\section{Disclosure}

The authors report no conflicts of interest in this work.

\section{References}

1. Ohno-Matsui K, Lai TYY, Lai -C-C, Cheung CMG. Updates of pathologic myopia. Prog Retin Eye Res. 2016;52:156-187. doi:10.1016/j.preteyeres.2015.12.001

2. Ruiz-Medrano J, Montero JA, Flores-Moreno I, Arias L, García-Layana A, Ruiz-Moreno JM. Myopic maculopathy: current status and proposal for a new classification and grading system (ATN). Prog Retin Eye Res. 2019;69:80-115.

3. Holden BA, Fricke TR, Wilson DA, et al. Global prevalence of myopia and high myopia and temporal trends from 2000 through 2050. Ophthalmology. 2016;123(5):1036-1042.

4. Xu L, Cui T, Yang H, et al. Prevalence of visual impairment among adults in China: the Beijing Eye Study. Am J Ophthalmol. 2006;141 (3):591-593.

5. Wong YL, Sabanayagam C, Ding Y, et al. Prevalence, risk factors, and impact of myopic macular degeneration on visual impairment and functioning among adults in Singapore. Invest Ophthalmol Vis Sci. 2018;59(11):4603-4613.

6. Iwase A, Araie M, Tomidokoro A, Yamamoto T, Shimizu H, Kitazawa Y. Prevalence and causes of low vision and blindness in a Japanese adult population: the Tajimi Study. Ophthalmology. 2006;113(8):1354-1362.

7. Klaver CC, Wolfs RC, Vingerling JR, Hofman A, de Jong PT. Agespecific prevalence and causes of blindness and visual impairment in an older population: the Rotterdam Study. Arch Ophthalmol. 1998;116(5):653-658.

8. Cedrone C, Nucci C, Scuderi G, Ricci F, Cerulli A, Culasso F. Prevalence of blindness and low vision in an Italian population: a comparison with other European studies. Eye (London, England). 2006;20(6):661-667.

9. Cotter SA, Varma R, Ying-Lai M, Azen SP, Klein R. Causes of low vision and blindness in adult Latinos: the Los Angeles Latino Eye Study. Ophthalmology. 2006;113(9):1574-1582.

10. Panozzo G. Optical coherence tomography findings in myopic traction maculopathy. Arch Ophthalmol. 2004;122(10):1455-1460. doi:10.1001/archopht.122.10.1455

11. Wu PC, Chen YJ, Chen YH, et al. Factors associated with foveoschisis and foveal detachment without macular hole in high myopia. Eye (London, England). 2009;23(2):356-361.

12. Johnson MW. Myopic traction maculopathy: pathogenic mechanisms and surgical treatment. Retina (Philadelphia, Pa). 2012;32(Suppl 2): S205-210.

Clinical Ophthalmology

\section{Publish your work in this journal}

Clinical Ophthalmology is an international, peer-reviewed journal covering all subspecialties within ophthalmology. Key topics include: Optometry; Visual science; Pharmacology and drug therapy in eye diseases; Basic Sciences; Primary and Secondary eye care; Patient Safety and Quality of Care Improvements. This journal is indexed on PubMed

Submit your manuscript here: https://www.dovepress.com/clinical-ophthalmology-journal
13. Morales MU, Saker S, Wilde C, Rubinstein M, Limoli P, Amoaku WM. Biofeedback fixation training method for improving eccentric vision in patients with loss of foveal function secondary to different maculopathies. Int Ophthalmol. 2020;40(2):305-312.

14. Duker JS, Kaiser PK, Binder S, et al. The International Vitreomacular Traction Study Group classification of vitreomacular adhesion, traction, and macular hole. Ophthalmology. 2013;120(12):2611-2619.

15. Ohno-Matsui K, Jonas JB. Posterior staphyloma in pathologic myopia. Prog Retin Eye Res. 2019;70:99-109.

16. Ye J, Shen M, Huang S, et al. Visual acuity in pathological myopia is correlated with the photoreceptor myoid and ellipsoid zone thickness and affected by choroid thickness. Invest Ophthalmol Vis Sci. 2019;60(5):1714-1723.

17. Qin Y, Zhu M, Qu X, et al. Regional macular light sensitivity changes in myopic Chinese adults: an MP1 study. Invest Ophthalmol Vis Sci. 2010;51(9):4451-4457.

18. Zaben A, Zapata M, Garcia-Arumi J. Retinal sensitivity and choroidal thickness in high myopia. Retina (Philadelphia, Pa). 2015;35 (3):398-406.

19. Alzaben Z, Cardona G, Zapata MA, Zaben A. Interocular asymmetry in choroidal thickness and retinal sensitivity in high Myopia. Retina (Philadelphia, Pa). 2018;38(8):1620-1628.

20. Wang Y, Ye J, Shen M, et al. Photoreceptor degeneration is correlated with the deterioration of macular retinal sensitivity in high Myopia. Invest Ophthalmol Vis Sci. 2019;60(8):2800-2810.

21. Cai L, Sun Z, Guo D, et al. Long-term outcomes of patients with myopic traction maculopathy after phacoemulsification for incident cataract. Eye (London, England). 2019;33(9):1423-1432.

22. Ikuno Y, Sayanagi K, Soga K, Oshima Y, Ohji M, Tano Y. Foveal anatomical status and surgical results in vitrectomy for myopic foveoschisis. Jpn J Ophthalmol. 2008;52(4):269-276.

23. Shimada N, Tanaka Y, Tokoro T, Ohno-Matsui K. Natural course of myopic traction maculopathy and factors associated with progression or resolution. Am J Ophthalmol. 2013;156(5):948-957.e941.

24. Wong CW, Phua V, Lee SY, Wong TY, Cheung CM. Is choroidal or scleral thickness related to myopic macular degeneration? Invest Ophthalmol Vis Sci. 2017;58(2):907-913.

25. Gupta P, Saw SM, Cheung CY, et al. Choroidal thickness and high myopia: a case-control study of young Chinese men in Singapore. Acta Ophthalmol. 2015;93(7):e585-592.

26. Jonas JB, Ohno-Matsui K, Spaide RF, Holbach L, Panda-Jonas S. Macular Bruch's membrane defects and axial length: association with gamma zone and delta zone in peripapillary region. Invest Ophthalmol Vis Sci. 2013;54(2):1295-1302.

27. Read SA, Fuss JA, Vincent SJ, Collins MJ, Alonso-Caneiro D. Choroidal changes in human myopia: insights from optical coherence tomography imaging. Clin Exp Optometry. 2019;102(3):270-285.

28. Entezari M, Karimi S, Ramezani A, Nikkhah H, Fekri Y, Kheiri B. Choroidal thickness in healthy subjects. J Ophthalmic Vis Res. 2018;13(1):39-43.

29. Gaucher D, Haouchine B, Tadayoni R, et al. Long-term follow-up of high myopic foveoschisis: natural course and surgical outcome. $\mathrm{Am}$ J Ophthalmol. 2007;143(3):455-462.
Central and CAS, and is the official journal of The Society of Clinical Ophthalmology (SCO). The manuscript management system is completely online and includes a very quick and fair peer-review system, which is all easy to use. Visit http://www.dovepress.com/ testimonials.php to read real quotes from published authors. 